\title{
Diagnostic problems with follicular thyroid cancer - case study
}

\author{
Monika Koziolek ${ }^{1 *}$, Anna Sieradzka ${ }^{1}$, Michal Jakuszewski ${ }^{2}$, \\ Lilianna Osowicz-KoroloneK ${ }^{1}$, Katarzyna Karpinska-KaczmarczyK ${ }^{3}$, Anhelli Syrenicz ${ }^{1}$
}

${ }^{1}$ Department of Endocrinology, Metabolic Diseases and Internal Diseases, Pomeranian Medical University in Szczecin, Unii Lubelskiej 1, 71-252 Szczecin, Poland

${ }^{2}$ Clinic of Orthopaedics and Traumathology, Pomeranian Medical University in Szczecin, Poland

${ }^{3}$ Department of Patomorphology, Pomeranian Medical University in Szczecin, Poland

\section{ARTICLE INFO}

Received 09 January 2014

Accepted 24 January 2014

\section{Keywords:}

minimally invasive follicular,

thyroid cancer,

bone metastasis.

\begin{abstract}
The diagnosis of follicular thyroid cancer is based on postoperative histopathology assessment. In its minimally invasive form, the signs of vascular invasion and capsular infiltration may sometimes be seen only in a small tumor fragment; hence, the diagnosis should be based on multiple histopathology specimens. This case study is a report on a 70-year-old female who was diagnosed with spinal metastasis of follicular thyroid cancer. This diagnosis was established 5 years after partial strumectomy due to goiter and there were no signs of thyroid cancer in postoperative histopathology assessment. Based on this case and literature reports, the authors conclude that the diagnosis of follicular thyroid cancer, especially its minimally invasive forms, may pose a diagnostic problem even when based on postoperative histopathology.
\end{abstract}

\section{INTRODUCTION}

Follicular thyroid neoplasms include follicular adenoma and follicular cancer. The ratio of follicular adenoma to follicular cancer of thyroid is 5 to 1 [9]. Presently, it is impossible to distinguish between these two tumors based on clinical, sonographic or cytologic features [9]. Follicular thyroid cancer, just like follicular adenoma, is usually a round, encapsulated tumor [5]. Cytology material shows cancer cells and adenoma cells forming follicular structures, without signs of atypia or mitotic figures. The diagnosis of follicular cancer is only possible in histopathology investigation. It requires the evidence for the infiltration of cancer cells through the entire capsule and/or vascular invasion [9,3]. Signs of vascular invasion and tumor extension through the entire capsule may sometimes be seen only in a small tumor fragment; therefore, correct diagnosis of minimally invasive cancer requires great precision and the review of multiple histopathology specimens sampled by experienced pathologist from a wholly removed tumor [4].

\footnotetext{
Corresponding author

* e-mail: koziolekm11@wp.pl

tel.: +4891425-3540

fax: $+4891425-3542$
}

We present a case of 70-year-old female with dissemination of the disease following diagnostic problems with minimally invasive follicular thyroid cancer.

\section{CASE STUDY}

A 70-year-old woman with the history of partial strumectomy in 2007 due to goiter confirmed by histopathology assessment was admitted to the Department of Orthopedics, Pomeranian Medical University in October 2012 because of pathological fracture of first lumbar vertebra.

Medical history included: hypertension, superficial phlebitis in lower limbs (March 2012), pulmonary embolism (April 2012), deep vein thrombosis in lower limbs and status after cholecystectomy. The patient was on chronic treatment with $100 \mu \mathrm{g} / \mathrm{d}$ levothyroxine, $4 \mathrm{mg} / \mathrm{d}$ acenocoumarol and $5 \mathrm{mg} / \mathrm{d}$ perindopril. On the day of admission, the patient complained about lumbar pain which has been getting stronger and stronger for 2 months; the pain had sudden onset and made it difficult for the patient to move.

Abnormal findings in physical examination included: obesity, BP160/100 mmHg, slight edema of both lower extremities, lower limb varices, scar after thyroid surgery and another scar after cholecystectomy.

Magnetic resonance imaging (MRI) of lumbosacral spine showed that the body of first lumbar vertebra (L1) produced abnormal, hypointensive signal. Within this signal, there was a pathological structure with the size of $34 \times 33 \times 23 \mathrm{~mm}$ which 
compressed the front part of dural sac. At this level, the width of spinal canal in sagittal cross-section was reduced to approx. $10.2 \mathrm{~mm}$ (relative stenosis), while it ranged from 17 to $19 \mathrm{~mm}$ at the remaining lumbar levels. MRI findings suggested secondary (metastatic) nature of the pathological tissue mass detected in L1 vertebral body. In order to find the primary malignant focus, chest $\mathrm{CT}$ with contrast enhancement was performed showing a 4-mm tumor in IX segment of left lung and pathological fracture of L1 vertebral body; at the level of L1, a protrusion of pathological lesion from vertebral body to spinal canal was observed, with enhancement after contrast administration. CT was followed by skeletal SPECT/CT with intravenous injection of $780 \mathrm{MBq}$ Tc99m-labelled MDP. Planar scintigram and CT cross-sections showed foci with enhanced radiomarker accumulation in the projection of right clavicle acromion and in L1 vertebra, while the rest of the skeleton had normal radiomarker distribution. A conclusion was made that the focus found in L1 vertebra corresponded to past fracture (most likely pathological fracture), while a lesion in the clavicle was probably of arthropathic nature. Ultrasound of abdomen did not show any significant anomalies. Having completed the diagnostic imaging, the patient was qualified for surgery (October 2012), which included decompression of spinal canal at the level of L1, removal of L1 vertebral body, insertion of vertebral body prosthesis and transpedicular stabilization of thoracolumbar spine.

Histopathological findings in L1 vertebral body included the metastatic lesion of follicular thyroid cancer. This diagnosis was confirmed with positive immunochistochemical reaction for the presence of thyroglobulin. In November 2012, the patient had ultrasound of thyroid which showed residual stump of right thyroid lobe, the stump of left thyroid lob - size $32 \times 15 \times 14 \mathrm{~mm}$ - with normoechogenic solid focal lesion of $7 \mathrm{~mm}$ diameter. Cervical lymph nodes were not enlarged. The patient was referred to the Department of Endocrine Surgery, Pomeranian Medical University, where she underwent total thyroidectomy with the removal of peritracheal and upper mediastinal lymph nodes as well as lateral cervical lymph nodes located on the left side (December 2012). Postoperative histopathology findings included goiter without features of malignancy and 11 reactive lymph nodes. As the diagnosis indicated a benign lesion of the thyroid and the patient had spinal metastasis of follicular cancer, histopathology of the material sampled from L1 vertebral body was re-assessed (January 2013), but the previous diagnosis (October 2012) was confirmed. The next step was to verify thyroid specimens obtained during the first surgery in 2007. Re-assessment of histopathology specimens produced the diagnosis of minimally invasive follicular cancer with the extension of cancer cells through the entire capsule and without vascular invasion. In February 2013, the patient had PET-CT with 18-F-fluorodeoxyglucose which showed a metabolically active lesion suggestive of metastasis to L1 vertebra and to the proximal part of right clavicle. The patient was qualified for radioactive iodine treatment and received $100 \mathrm{mCi} \mathrm{I} 131$ isotope. Post-treatment iodine scintigraphy of the whole body showed excessive radiomarker uptake in $1^{\text {st }}$ lumbar vertebra, in medial part of right clavicle and in the thyroid bed, which was more intensive on the right side.

\section{DISCUSSION}

Follicular thyroid cancer is a highly differentiated malignant neoplasm originating from follicular cells. It accounts for approx. 10-15\% thyroid cancers [5,3]. Based on prognostic factors, follicular thyroid cancer is divided into encapsulated form and the form with massive invasion. Encapsulated follicular cancer may only be associated with capsule invasion and occasionally with vascular invasion (involving less than 4 vessels), or with extensive vascular invasion (involving 4 or more vessels) [11]. In patients with correct diagnosis and appropriate treatment of minimally invasive encapsulated follicular cancer, 10-year survival rate ranges from $100 \%$ (cases without vascular invasion) to $70 \%$ (patients with extensive vascular invasion). The survival rate is reduced to $25-45 \%$ in patients with follicular cancer and massive invasion [11]. Follicular cancer is spread mainly through blood, producing remote metastases, chiefly to lungs and bones [10]. The prognosis is worse for patients with bone metastases of follicular cancer than for those with lung metastases [6]. Metastases of follicular thyroid cancer are more common in tumors with extensive vascular invasion, they are rare - about 5\% - in minimally invasive tumors with vascular infiltration and they occur in only $1 \%$ tumors diagnosed as minimally invasive follicular cancer based on minimal invasion of the capsule [11].

Bernier M. et al. analyzed a group of patients with bone metastases of differentiated follicular thyroid cancer. Histopathology assessment of thyroid tumor yielded a diagnosis of follicular cancer in 77 patients and papillary cancer in 19 patients. The remaining 12 patients had a history of partial thyroidectomy which took place 1-42 years before the diagnosis of bone metastasis of differentiated thyroid cancer and, just as in our case, the cancer was not diagnosed based on histopathology of thyroid tumor. Unfortunately, the authors did not provide details on how many patients had the metastasis of follicular cancer and how many of them were diagnosed with the metastasis of papillary thyroid cancer [2]. On the other hand, Ban E. et al. presented a group of 10 patients with remote metastases of follicular thyroid cancer. Histopathology assessment of thyroidectomy material revealed minimally invasive cancer in 4 patients and widely invasive cancer in 3 subjects. The remaining 3 patients did not have features suggestive of follicular cancer in the histopathology investigations of thyroid. Re-evaluation of histopathology of thyroid specimens from those 3 patients performed once the remote metastases have been detected, produced the following diagnoses: follicular lesion with features favoring hyperplastic nodule, without features of neoplasia; single atypical adenoma without features of malignancy; multi-nodular thyroid parenchyma with multiple hyperplastic nodules and extensive fibrosis. The authors fail to report whether thyroid tumors were investigated as a whole and how many specimens were sampled for histopathology assessment [1]. Lack of evidence for follicular cancer in thyroid specimens obtained from 
those 3 patients may have resulted from insufficient number of specimens sampled from the tumors, which is particularly significant in minimally invasive cancer. This problem has also been raised by Huang $C$. et al. who have pointed out that improving the rate of correct diagnosis of follicular thyroid cancer is related to increasing the number of specimens sampled from postoperative material [7]. Lin J. et al. who investigated a group of 70 patients with lung metastases of follicular thyroid cancer emphasized that one of the reasons for advanced stage of cancer at the time of diagnosis is initial misdiagnosis of benign thyroid tumor in patients with follicular cancer [8], which was also the case in our patient.

Our case as well as literature reports highlight the possibility of remote metastases, even in minimally invasive follicular thyroid cancer and raise diagnostic problems with follicular cancer, particularly its minimally invasive form, even based on histopathology assessment. It should be stressed again that features of malignancy indicating minimally invasive follicular thyroid cancer are sometimes detectable only in a small tumor fragment. Therefore, correct diagnosis requires the assessment of many histopathology specimens from the tumor removed as a whole, sampled with particular precision and by an experienced pathologist.

\section{CONCLUSION}

The diagnosis of follicular thyroid cancer, especially its minimally invasive forms, may pose a problem, even based on postoperative histopathology assessment.

\section{REFERENCES}

1. Ban E.J. et al.: Follicular thyroid cancer: minimally invasive tumors can give rise to metastases. ANZ J Surg., 82(3), 137-138, 2012.

2. Bernier M.O. et al.: Survival and therapeutic modalities in patients with bone metastases of differentiated thyroid carcinomas. J Clin Endocrinol Metab., 86(4), 1569, 2001.

3. D'Avanzo A. et al.: Follicular thyroid carcinoma: histology and prognosis. Cancer., 100(6), 1124, 2004.

4. Goldstein N.S., Czako P., Neill J.S.: Metastatic minimally invasive follicular and Hurthle cell thyroid carcinoma. Mod Pathol., 13, 128, 2000.

5. Heffess C.S., Thompson L.D.: Minimally invasive follicular thyroid carcinoma. Endocrine Pathol., 1, 12, 417, 2001.

6. Hindié E. et al.: Bone metastases of differentiated thyroid cancer: impact of early 131I-based detection on outcome. Endocr Relat Cancer.,14(3), 800, 2007.

7. Huang C.C. et al.: Diagnostic and therapeutic strategies for minimally and widely invasive follicular thyroid carcinomas. Surg Oncol., 20(1), 4, 2011.

8. Lin J.D., Chao T.C., Hsueh C.: Follicular thyroid carcinomas with lung metastases: a 23-year retrospective study. Endocr J., 51(2), 223, 2004.

9. McHenry C.R., Phitayakorn R.: Follicular adenoma and carcinoma of the thyroid gland. Oncologist., 16(5), 586-588, 2011.

10. Qiu Z.L. et al.: Efficacy and survival analysis of 131I therapy for bone metastases from differentiated thyroid cancer. J Clin Endocrinol Metab., 96(10), 3078, 2011.

11. Stephenson T. (2013). Patologia chorób tarczycy. In: Choroby tarczycy i przytarczyc. Praktyczny podręcznik. Lewiński A. (editor). Warszawa: MediPage; p. 21-22. 